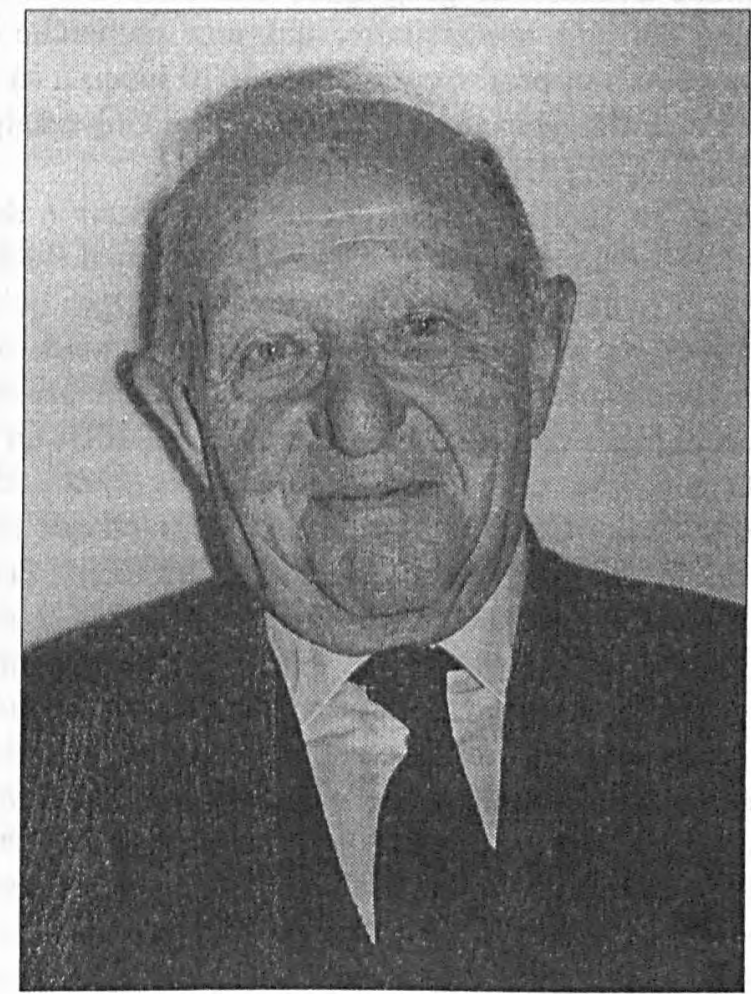

\title{
CURRICULUM VITAE DE BERNARD BARBIER
}

Il est né le 4 mai 1927, cinquième d'une famille de six enfants; son père était officier de marine. Il vit dans un appartement situé dans l'Ile Saint-Louis (rue de Bretonvilliers, Paris, IV ${ }^{\text {ème }}$ arrondissement), qui est dans la famille de sa mère depuis 1824; très jeune, il entend parler de la Pologne, car certaines fenêtres de l'appartement donnent sur l'Hôtel Lambert, rue Sant-Louis en L'Ile, où vivait encore le Pronce Czartoryski, et ses parents ne manquent pas de lui montrer la Bibliothèque Polonaise, Quai d'Orléans, ou bien l'immeuble où vécut Maria Skłodowska-Curie, Quai de Béthune. Il est élève à l'Ecole Massillon, tenue par des Oratoriens, puis au Lycée Louis Le Grand, où il passe ses deux baccalauréats.

Il devient étudiant à la Sorbonne, où il fait de l'historie et surtout de la géographie; il y suit les cours des grands maîtres de l'époque: le Doyen A. Cholley et surtout le Professeur G. Chabot, qui connaissait bien la Pologne. En 1949, il est reçu à l'agrégation de géographie. Il part effectuer son service militaire, qu'il termine comme aspirant d'artillerie sur les rives du Lac de Constance. Il a, d'ailleurs, toute sa vie, été intéressé par l'art de la guerre, ainsi que par l'histoire militaire: il est reçu, en 1956, au concours de l'Ecole d'Etat-Major pour Officiers de Réserve et termine sa vie militaire comme lieutenant-colonel honoraire.

Pour le principe, il avait été nommé au Lycée de Pontivy (Bretagne) pendant la durée de son service, mais il n'y a jamais enseigné. Revenu à la vie civile, il est nommé au Lycée de Gap (Hautes-Alpes), en octobre 1950, puis à celui d'Annecy (Haute-Savoie) l'année suivante.

En 1952, il se marie avec Madeleine Brossat, qui avait enseigné avee lui à Gap et qui fera toute sa carrière comme astronome à l'Observatoire de Marseille, ainsi qu'à celui de Saint-Michel l'Observatoire. Quatre enfants vont naître, entre 1954 et 1965, dont un va choisir le métier d'enseignant (en géographie); les trois autres sont: éducatrice spécialisée, chirurgien-dentiste et cadre de banque. Il s'installe donc à Marseille et est nommé à l'Ecole de l'Air (Salon-de-Provence), où il fait des cours aux élèves-officiers sur les grands problèmes géopolitiques et économiques du monde. Il enseigne également à l'Ecole Supérieure de Commerce de Marseille (1958-1969) et il sera même président du jury des épreuves de géographie économique au concours national d'entrée aux Ecoles de Commerce. 
En octobre 1960, il est nommé assistant de géographie à la Faculté des Lettres d'Aix en Provence, choisissant alors de mener une carrière universitaire, qui unit recherche et enseignement. Il y est successivement maître-assistant (1962) et professeur, depuis 1970 jusqu'à sa retraite, à l'exception d'un bref intermède où il est maître de conférences au tout jeune Centre Universaire d'Avignon (1969-1970). Depuis 1992 il est Professeur Emérite de l'Université de provence.

Pour sa thèse d'Etat, soutenue en 1969, il choisit d'étudier la question du réseau urbain en pays de montagne, dans les Alpes du Sud plus exactement; depuis 1942, où, à l'occasion d'un camp scout en Savoie, il découvre la montagne et la neige, il est passionné par les Alpes qu'il étudiera et visitera toute sa vie. Les domaines de son travail, en géographie générale, sont, d'abord, celui du rôle des villes dans l'organisation et l'aménagement de l'espace; ses collègues le portent à la présidence de la Commission de Géographie Urbaine (Comité National Français de Géographie), fonction qu'il occupe de 1974 à 1980. Mais, c'est la géographie du tourisme qui constitue le premier centre de ses préoccupations (cf. Bibliographie) et, à Tokyo, en 1980, au Congrès de l'Union Géographique Internationale (U.G.I.), il est élu président de la Commission de Géographie du tourisme et des loisirs de cette U.G.I.; il abandonne cette responsabilité en 1988, mais il l'occupe en fait jusqu'en 1992. Il est membre de l'A.I.E.S.T. (Association Internationale des Experts Scientifiques du Tourisme), de 1969 jusqu'en 1999. Cependant, il n'imagine que l'on puisse faire de la géographie sans mener des recherches sur des espaces régionaux et il pense, encore aujourd'hiu, que la "régionale" reste une approche fondamentale de la discipline. S'il visite le plus de pays possible à l'occasion de congrès ou de voyages touristiques, il les étudie "livresquement" au préalable et il les regarde ensuite en géographie, afin de les comprendre et les connaître, tout en prenant beaucoup de diapositives pour ses cours ou des conférences ultérieures. C'est ainsi que, à côté du monde provençal et alpin, il s'intéresse aux pays du Maghreb, notamment au Maroc: il reçoit beaucoup d'étudiants de ces pays et dirige trente thèses sur ce seul Maroc. Un autre espace le passionne, celui de l'Europe centre-orientale; il y fait son premier voyage en 1966, menant des étudiants en Tchécoslovaquie, et il y fera quelque soixante-dix missions ou séjours. Toutefois, c'est la Pologne qui "l'accroche" le plus et il s'y rend trente-cinq fois. Désireux de nouer des liens scientifiques avec ce pays et de bien l'étudier, il veut établir des conventions avec des Universités polonaises. Il passe la première, en 1974, avec l'Institut de Géographie économique de Łódź (Professeur L. Straszewicz), qu’il a le plasir de signer lui-même, car il est alors Vice-Président de son Université; en 1981, il conclut un nouvel accord, avec l'Institut de Géographie de l'Académie des Sciences de Pologne (Professeurs J. Kostrowicki et M. Rościszewski). Ces deux conventions vont durer vingt ans et se traduire par des échanges d'enseignants (et même d'étudiants), des colloques communs et des publications. Les géographes polonais lui font l'honneur de l'élire Membre d'Honneur de la Société Polonaise de Geographie, en 1973. L'Université de Łódź lui décerne le titre de Docteur honoris causa, en 1990.

Le métier de "professeur" comporte beausoup de tâches qui ne relèvent pas directement de la recherche ou de l'enseignement. Il est membre du jury du concours de l'agrégation (1972-1974), puis du concours d'entrée à l'Ecole Normale Supérieure de Fontenay-aux-Roses. Il est élu au Comité National Français de Géographie (1976-1984), ce qui lui vaut de participer à la préparation du Congrès de l'U.G.I. à Paris, en 1980, et d'y inviter beaucoup de Polonais, qui s'y rendent malgré les difficultés politiques de l'époque. Il reste près de dix ans au Comité Consultatif des Universités (1973-1977 et 1979-1983), organisme officiel d'élus qui, sous des vocables successifs (C.C.U., C.S.C.U., C.N.U.), a la responsabilité de gérer la carrière des enseignants du Supérieur, de vérifier le bien-fondé des élections dans les universités et d'apprécier, en dernier ressort, la qualité des thèses de doctorat; cette fonction est délicate, mais très intéressante, car elle place son titulaire au cœur de la recherche et de la vie des universités.

A côté de son travail universitaire, il s'occupe de la Société de Géographie de Marseille, dont il est président depuis 1985. Il est élu membre de l'Académie de Marseille en 1990 et il en est le Chancelier, en 1996, puis le Directeur, en 1997. Sensible à l'intérêt porté par un très grand nombre à la culture et à la langue françaises, il accepte volontiers de faire, presque chaque année, des tournées de conférences à l'étranger pour l'Alliance Française; le premier pays visité est d'ailleurs la Pologne, en 1973, et le dernier les Etats-Unis en 2001.

Il est Chevalier dans l'Ordre National du Mérite, Titulaire de la Médaille d'argent des Services Militaires Volontaires, Chevalier des Palmes Académiques. 